Pesq. Vet. Bras. 30(9):763-769, setembro 2010

\title{
Padronização do eletrorretinograma de campo total em cães ${ }^{1}$
}

\author{
Angélica M.V. Safatle ${ }^{2 *}$, Ana Paula Hvenegaard², Márcia Kahvegian ${ }^{3}$, Teresinha \\ L. Martins ${ }^{3}$, Graziele M. Shimamura ${ }^{2}$, Denise T. Fantoni ${ }^{2}$ e Paulo S.M. Barros ${ }^{2}$
}

\begin{abstract}
Safatle A.M.V., Hvenegaard A.P., Kahvegian M., Martins T.L., Shimamura G.M., Fantoni D.T. \& Barros P.S.M. 2010. [Full field electrorretinogram standartization in dogs.] Padronização do eletrorretinograma de campo total em cães. Pesquisa Veterinária Brasileira 30(9):763-769. Departamento de Cirurgia, Faculdade de Medicina Veterinária e Zootecnia, Universidade de São Paulo, 05508-270 São Paulo, SP, Brazil. E-mail: angsaf@usp.br

Electroretinogram (ERG) is an objective non invasive diagnostic method to evaluate retinal function which permits the early detection of lesions at retinal external layers, even before the appearance of clinical signs. In dogs, ERG is mostly utilized for preoperative evaluation in patients presenting cataracts; characterization of disturbances causing blindness, among the utilization of dogs as animal models in scientific research. Several factors interfere in ERG responses, such as the ERG machine, light stimulation, electrode, time spent on dark adaptation, pupil size, means opacity, sedation or anesthetization, species, breed and age. The purpose of this study was to standardize the full field ERG in sedated dogs, according to the International Society for Clinical Electrophysiology of Vision (ISCEV) protocol, using Ganzfeld and bipolar electrodes. Two hundred thirty threes ERGs were performed in 147 female and 86 male with ages from 1 to 14 years old. Among those 233 dogs, 100 presented cataracts in different stages of maturation, 72 were diabetic and presented mature or hypermature cataracts, 26 presented ERG compatible to progressive retinal degeneration, 3 presented ERG compatible to sudden acquired retinal degeneration syndrome; for 32 dogs no abnormal ERG was registered and they were considered as having normal retinal function. Sedation was capable to induce a good immobilization with no bulb rotation, resulting uniform retinal stimulation, using Ganzfeld. Veris system successfully registered all 5 ISCEV responses, simultaneously from both eyes, at the same time. Full field ERG became a fundamental ophthalmic exam, then, its standardization is mandatory to allow comparison between ERGs from different laboratories. The reliability and reproducibility of this protocol has shown very good responses, using ISCEV protocol, Veris system, Ganzfeld and Burian Allen electrodes in sedated dogs.
\end{abstract}

INDEX TERMS: Dog, retina, full field electroretinogram.

RESUMO-- Eletrorretinograma (ERG) é o meio diagnóstico objetivo e não-invasivo para avaliar a função retiniana e detectar precocemente, em várias espécies, lesões nas suas camadas mais externas. As indicações mais comuns para ERG em cães são: avaliação pré-cirúrgica de pacientes com

\footnotetext{
${ }^{1}$ Recebido em 23 de fevereiro de 2010.

Aceito para publicação em 10 de junho de 2010.

2 Departamento de Cirurgia, Faculdade de Medicina Veterinária e Zootecnia (FMVZ), Universidade de São Paulo (USP), Av. Prof. Dr. Orlando Marques de Paiva 87, Bloco 8 Superior, Cidade Universitária, São Paulo, SP 05508-270, Brasil. *Autor para correspondência: angsaf @usp.br

${ }^{3}$ Pós-Graduanda do Programa de Pós-Graduação de Anestesiologia, Faculdade de Medicina (FM), Universidade de São Paulo (USP), Av. Dr. Arnaldo 455, Cerqueira César, São Paulo, SP 01246-903.
}

catarata, caracterização de distúrbios que causam cegueira, além de servir como importante modelo para o estudo da distrofia retiniana que acomete o homem. Vários são os fatores que podem alterar o ERG tais como: eletrorretinógrafo, fonte de estimulação luminosa, tipo do eletrodo, tempo de adaptação ao escuro, tamanho pupilar, opacidade de meios e protocolo de sedação ou anestesia; além da espécie, raça e idade. Objetivou-se com este estudo padronizar o ERG para cães submetidos à sedação, seguindo o protocolo da International Society for Clinical Electrophysiology of Vision (ISCEV), utilizando Ganzfeld e eletrodos Burian Allen. Foram realizados 233 eletrorretinogramas em cães, 147 fêmeas e 86 machos, com idades entre um e 14 anos. Dos 233 cães examinados, 100 apresentavam catarata em diferentes estágios de maturação, 72 eram diabéticos e apresentavam cata- 
rata madura ou hipermadura, 26 apresentaram eletrorretinograma compatível com degeneração retiniana progressiva, três apresentaram eletrorretinograma compatível com síndrome da degeneração retiniana adquirida subitamente e 32 não apresentaram lesão retiniana capaz de atenuar as respostas do ERG, sendo considerados normais quanto à função retiniana. A sedação foi capaz de produzir boa imobilização do paciente sem rotacionar o bulbo ocular, permitindo adequada estimulação retiniana bilateralmente, com auxílio do Ganzfeld. O sistema eletrodiagnóstico Veris registrou com sucesso e simultaneamente de ambos os olhos, as cinco respostas preconizadas pela ISCEV. Como o ERG de campo total tornouse exame fundamental na rotina oftalmológica, sua padronização é indispensável quando se objetiva comparar resultados de laboratórios distintos. A confiabilidade e reprodutibilidade deste protocolo foi demonstrada com a obtenção de registros de ótima qualidade utilizando protocolo padrão da ISCEV, eletrorretinógrafo Veris, Ganzfeld e eletrodos Burian Allen nos cães submetidos à sedação.

TERMOS DE INDEXAÇÃO: Cães, retina, eletrorretinograma de campo total.

\section{INTRODUÇÃO}

Eletrorretinograma (ERG) é o meio diagnóstico objetivo (Tzekov \& Arden 1999) e não-invasivo (Safatle et al. 2005) para avaliar a função retiniana e para detectar, precocemente, lesões em suas camadas mais externas (Komaromy et al. 1998). Por ser considerado um método de diagnóstico preciso (Komaromy et al. 1998), tem várias aplicações tanto na medicina humana (Birch 1989, Birch \& Anderson 1992, Paranhos et al. 1997, Català \& Castany 2005) como na medicina veterinária. $\mathrm{Na}$ medicina veterinária tem grande importância na clínica de pequenos animais, com aplicação também em grandes animais e animais exóticos (Rubin 1971, Hurn et al. 2003, Sims 1999, Komaromy et al. 2003, Narfström 2006, PetersenJones et al. 2006, Hendrix \& Sims 2004).

Em cães, as indicações mais comuns para ERG são: avaliação pré-cirúrgica de pacientes com catarata, caracterização de distúrbios que causam cegueira como glaucoma, acromatopsia (Rubin 1971, Hurn et al. 2003), displasia retiniana, retinopatias degenerativas (Safatle et al. 2005), hipoplasia de nervo óptico, síndrome da degeneração retiniana adquirida subitamente (SARDS) e lipofuscinose ceróide neuronal (Narfström et al. 2007), além de servir como importante modelo para o estudo da distrofia retiniana que acomete o homem (Petersen-Jones et al. 2006).

Aparelhos utilizados para a realização do ERG apresentam dois componentes principais: o sistema de estimulação e o de gravação. O sistema de estimulação é variável, podendo ser produzido por flash de luz difusa, por estimulação padrão ou por estimulação focal (Tzecov \& Arden 1999).

Para registrar a resposta ao estímulo luminoso, necessitam-se de três eletrodos: ativo, referência e terra. Eletrodos bipolares possuem o ativo e o referência juntos. Existem diversos modelos que podem ser utilizados tanto na medicina humana como na medicina veterinária, tais como:
ERG-jet, Burian Allen, Goldlens, bipolar com fonte luminosa acoplada e DTL (Hérbert et al. 1996, Maehara et al. 2005, Beeler et al. 2007). Ao compará-los, podemos observar que as respostas dos testes eletrofisiológicos diferem entre si (Petersen-Jones et al. 2006). O registro é composto por três ondas básicas: $A, B$ e $C$. A onda A é negativa. Logo em seguida há uma onda positiva, maior e mais lenta, denominada onda B. Geralmente se produz uma terceira onda, onda $C$, muito prolongada, seguida por um potencial final, designado de onda $D$. A onda A pode ser explicada como componente inicial córneo-negativo que reflete a atividade de transdução dos fotorreceptores (Gum 1980, Salomão 2002). A onda B é uma onda córneo-positiva de grande amplitude (Gum 1980) originária das células de Müller e bipolares (Dantas 1995). A onda C, que é o segundo pico positivo, aparece após um a dois segundos após o flash e está relacionada com a polarização do epitélio pigmentário (Gum 1980), que aparece após a onda B em olhos adaptados ao escuro, mas é pouco utilizada clinicamente (Kommonen \& Raitta 1987).

Para avaliar-se isoladamente cones e bastonetes, podese utilizar estímulos repetitivos ou variáveis, de maior ou menor intensidade, frequências de luzes oscilatórias (flickers), além da adaptação à luz ou ao escuro.

Em razão da grande variedade de protocolos utilizados para a execução do exame e de vários fatores que possam alterá-lo, em 1989 a International Society for Clinical Electrophysiology of Vision (ISCEV), juntamente com a Foundation Fighting Blindness, recomendaram um protocolo padrão internacional para o uso clínico do ERG de campo total (full-field ERG) no homem, que foi atualizado em anos subsequentes (Marmor \& Zrenner 1999, Marmor et al. 2004), com o objetivo de uniformizar a interpretação dos registros eletrorretinográficos feitos em diferentes locais (Marmor et al. 1989).

De acordo com a ISCEV (Marmor et al. 2004), as cinco respostas obtidas pelo exame são: resposta escotópica obtida pelos bastonetes, resposta escotópica máxima, potencial oscilatório, resposta fotópica obtida pelos cones e a resposta a estímulos repetitivos rápidos (flickera $30 \mathrm{~Hz}$ ).

O objetivo deste estudo foi padronizar o eletrorretinograma de campo total para os cães submetidos à sedação, utilizando o protocolo padrão da ISCEV com auxílio do Ganzfeld, eletrorretinógrafo Veris e eletrodos Burian Allen.

\section{MATERIAL E MÉTODOS}

Foram realizados 233 eletrorretinogramas em cães de diferentes raças, sendo 147 fêmeas e 86 machos, com idades variando entre um e 14 anos, encaminhados ao Serviço de Oftalmologia do Hospital Veterinário da Faculdade de Medicina Veterinária e Zootenia da Universidade de São Paulo, no período de Fevereiro de 2006 e Outubro de 2008, após aprovação da Comissão de Bioética da mesma faculdade (Protocolo no 06/2001).

O exame foi realizado em sala escura, com o paciente sob sedação. Realizou-se administração de sulfato de atropina ${ }^{4}$

\footnotetext{
${ }^{4}$ Atropion, Ariston Ind. Quím. e Farmacêuticas, Rua Adherbal Stresser 84, Jardim Arpoador, São Paulo, SP.
} 
(0,044 mg/kg, via SC). Instilou-se em ambos os olhos colírio de tropicamida $1 \%^{5}$, seguido de colírio de fenilefrina $10 \%{ }^{6}$, com intervalo de cinco minutos entre eles. A adaptação ao escuro foi feita com uso de bandagem oclusiva ${ }^{7}$ e teve duração de 30 minutos. Para sedação, foram administrados: cloridrato de xilazina $^{8}\left(0,5 \mathrm{mg} / \mathrm{kg}\right.$, via IM) e butorfanol ${ }^{9}(0,2 \mathrm{mg} / \mathrm{kg}$, via IM).

Com intuito de provocar acinesia palpebral, realizou-se o bloqueio do nervo palpebral após administração de cloridrato de lidocaína $2 \%{ }^{10}$ (4 mg/kg, via SC) no canto temporal à rima palpebral de ambos os olhos. Para obtenção dos registros, utilizou-se eletrodos bipolar Burian Allen ${ }^{11}$ preenchido com metilcelulose $2 \%{ }^{12}$, a fim de oferecer melhor contato elétrico e proteger a superfície corneal (Fig.1). Colírio anestésico ${ }^{13}$ foi administrado antes da colocação dos eletrodos. O eletrodo-terra, tipo ear-clip ${ }^{14}$, preenchido com creme eletrolítico ${ }^{15}$ foi colocado na borda auricular esquerda tricotomizada. Com os eletrodos devidamente colocados, posicionou-se o animal em decúbito

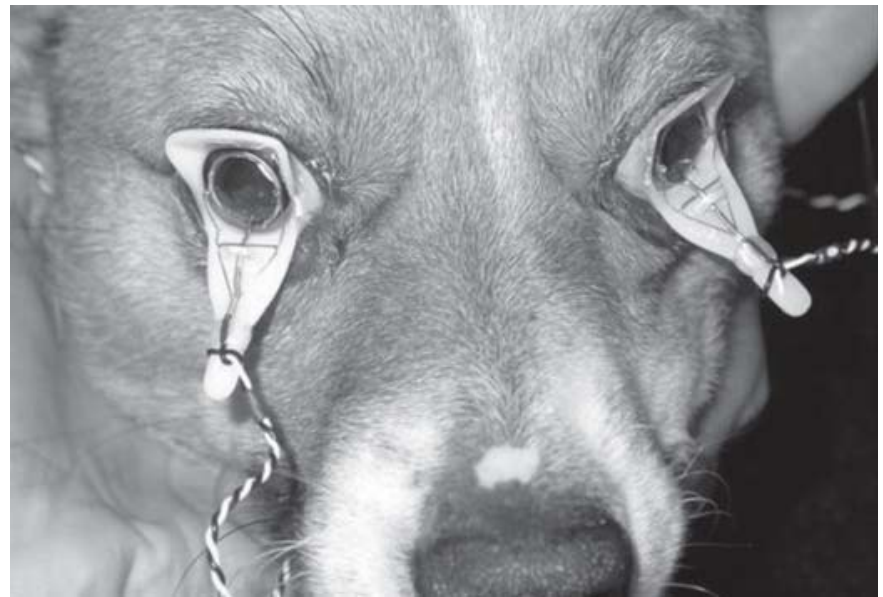

Fig.1. Eletrodo bipolar Burian Allen colocado sobre a córnea do cão submetido ao eletrorretinograma de campo total.

ventral, com a cabeça inteiramente colocada no interior da cúpula de estimulação: Ganzfeld ${ }^{16}$ (Fig.2). Observava-se com frequência a centralização dos olhos. Caso estivessem rotacionados ou apresentassem protrusão da terceira pálpebra, o paciente era estimulado com sinais sonoros.

Para a realização do ERG de campo total, utilizou-se o sistema eletrodiagnóstico Veris ${ }^{17}$, no qual um programa de computador comandava o número de apresentações e a duração dos estímulos luminosos. O intervalo interestímulos variou de um a 15 segundos.

\section{Protocolo-padrão humano (ISCEV)}

Registraram-se inicialmente as respostas escotópicas após adaptação ao escuro por 30 minutos e a seguir, acendia-

\footnotetext{
${ }^{5}$ Mydriacyl, Alcon Laboratórios do Brasil Ltda, Av. Nossa Senhora Assunção 736, Butantã, São Paulo, SP.

${ }^{6}$ Fenilefrina 10\%, Allergan Produtos Farmacêuticos Ltda, Av. Guarulhos 3180, Guarulhos, SP.

7 Atadura de Crepon, Neve Ind. e Com. Produtos Cirúrgicos, Rua Júlio Parigot 353, Antonieta, São Paulo, SP.

${ }^{8}$ Xilazin, Syntec do Brasil Ltda, Rua Soluções do Lar 105, Cotia, SP.

9 Torbugesic, Fort Dodge Saúde Animal Ltda, Rua Luiz Fernando Rodriguez 1701, Boa Vista, Campinas, SP.

${ }^{10}$ Xylestesin 2\%, Cristália Produtos Químicos e Farmacêuticos Ltda, Av. Paoletti 363, Itapira, SP.
}

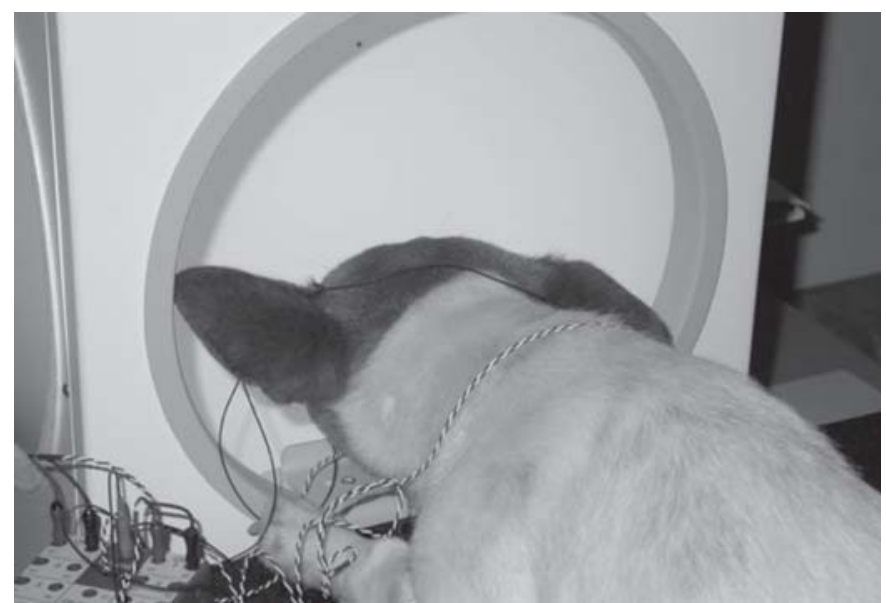

Fig.2. Animal em decúbito ventral e com a cabeça inteiramente colocada no interior da cúpula de estimulação (Ganzfeld).

se uma luz com $30 \mathrm{~cd} / \mathrm{m}^{2}$ de intensidade, gerada pelo próprio Ganzfeld, pelo período de 10 minutos, de modo a saturar os bastonetes e isolar as respostas dos cones (adaptação ao claro), sendo então registradas as respostas fotópicas ao flash único e posteriormente as respostas obtidas com estimulação intermitente (flicker) a uma frequência de $30 \mathrm{~Hz}$. Os registros foram realizados na sequência a seguir: resposta escotópica de bastonetes, resposta escotópica máxima, potenciais oscilatórios, resposta fotópica de cones obtida por flash único e flicker a $30 \mathrm{~Hz}$.

Em cada uma das cinco respostas do protocolo padrão da ISCEV, foram feitas 20 apresentações dos estímulos luminosos, sendo considerada como resposta final a média destes registros, exceto a resposta de flicker que foi resultante de uma

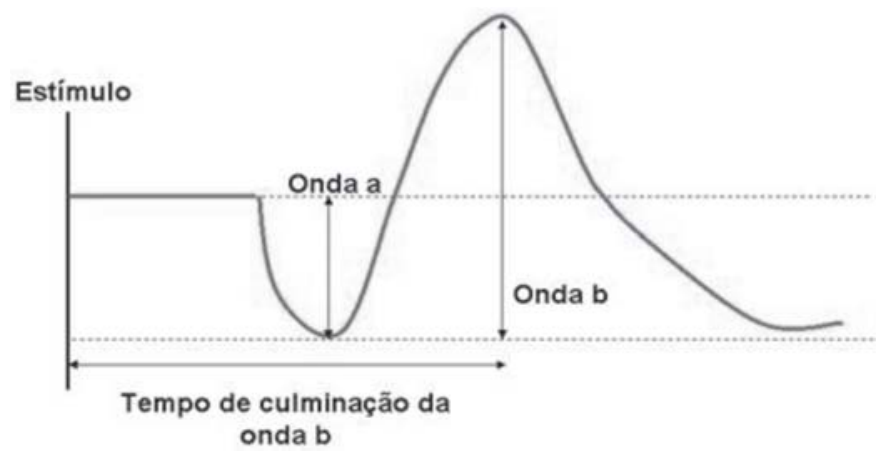

Fig.3. Esquema representativo da mensuração da amplitude das ondas a e b no eletrorretinograma de campo total.

\footnotetext{
${ }^{11}$ Burian Allen, Hansen Ophthalmic, 745 Avalon Place, Coralville, IA 52241, USA.

12 Metilcelulose 2\%, Ophthalmos Indústria Farmacêutica, Rua Nhandirobas 471, São Paulo, SP.

${ }^{13}$ Anestalcon, Alcon Laboratórios do Brasil Ltda, Av. Nossa Senhora Assunção 736, Butantã, São Paulo, SP.

${ }^{14}$ Gold Disc Electrode in Ear Clip, Grass Instrument Division, Astro Med., West Warwick, RI 02893, USA.

${ }^{15}$ Pasta Disk-Fix, Meditron Eletromedicina Ltda, Rua Alexande Finta 99, São Paulo, SP.

${ }^{16}$ Ganzfeld Stimulator, Electro-Diagnostic Imaging, 200F Twin Dolphin Dr., Redwood City, CA 94065-1402.

17 Veris System, Electro-Diagnostic Imaging, 200F Twin Dolphin Dr., Redwood City, CA 94065-1402, USA
} 


\section{Quadro 1. Protocolo do eletrorretinograma de campo total em cães}

\begin{tabular}{|c|c|}
\hline Condição & Procedimento \\
\hline Midríase & $\begin{array}{l}\text { Instilou-se colírio de tropicamida } 1 \% \text {, seguido de colírio de fenilefrina } 10 \% \text { com intervalo de cinco minutos en- } \\
\text { tre eles em ambos os olhos. }\end{array}$ \\
\hline Preparação para sedação & Administrou-se atropina $(0,044 \mathrm{mg} / \mathrm{kg})$ por via subcutânea. \\
\hline Sedação & Administrou-se, por via intramuscular, xilazina $(0,5 \mathrm{mg} / \mathrm{kg})$ e butorfanol $(0,2 \mathrm{mg} / \mathrm{kg})$. \\
\hline Bloqueio aurículo palpebral & Administrou-se lidocaína $2 \%(4 \mathrm{mg} / \mathrm{kg})$ no canto temporal à rima palpebral em ambos os lados. \\
\hline Colocação dos eletrodos & $\begin{array}{l}\text { Colocou-se o eletrodo Burian Allen sobre a córnea após preenchê-lo com metilcelulose } 2 \% \text {. Colocou-se o } \\
\text { eletrodo terra (ear-clip) no bordo auricular esquerdo tricotomizado e preenchido } \\
\text { com creme eletrolítico. }\end{array}$ \\
\hline Posicionamento do paciente & $\begin{array}{l}\text { Posicionou-se o cão em decúbito ventral e com a cabeça inteiramente colocada no interior da cúpula de esti- } \\
\text { mulação (Ganzfeld). }\end{array}$ \\
\hline Condição escotópica & $\begin{array}{l}\text { Registraram-se as respostas: resposta escotópica de bastonetes, resposta escotópica máxima e potenciais } \\
\text { oscilatórios. }\end{array}$ \\
\hline Acomodação ao claro & Acendeu-se uma luz de fundo com intensidade de $30 \mathrm{~cd} / \mathrm{m}^{2}$ pelo período de 10 minutos. \\
\hline Condição fotópica & $\begin{array}{l}\text { Registrou-se a resposta fotópica de cones obtida por flash único e, logo após, a respostas obtidas com esti- } \\
\text { mulação intermitente (flicker) a uma frequência de } 30 \mathrm{~Hz} \text {. }\end{array}$ \\
\hline
\end{tabular}

\section{Erg Campo Total}
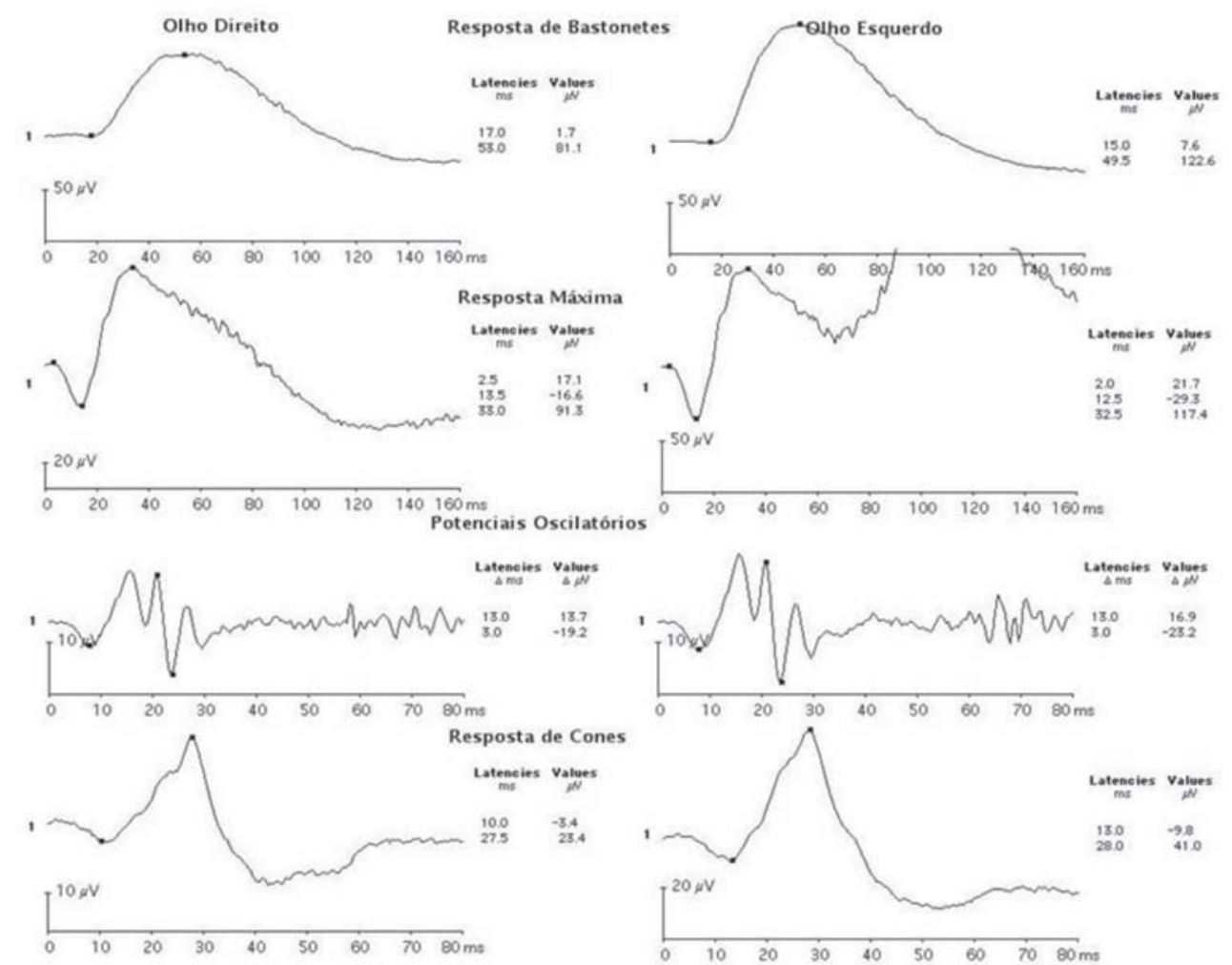

esposta de Cones

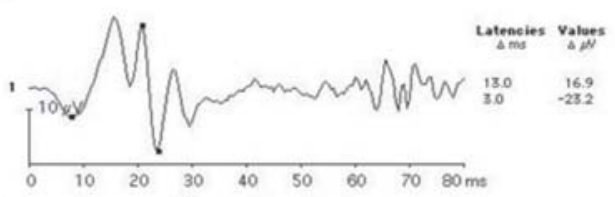
Field vier
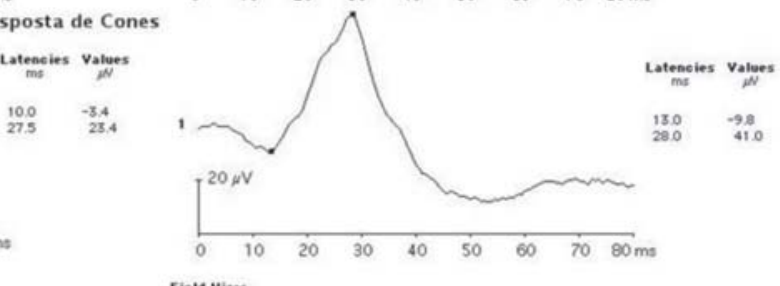

Flicker $30 \mathrm{~Hz}$
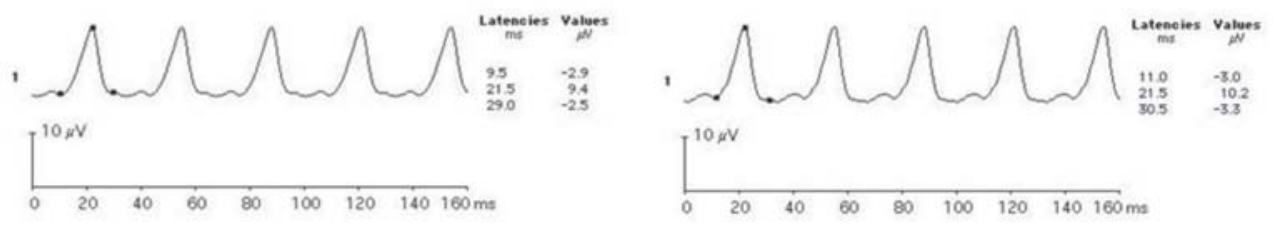

Fig.4. Modelo de eletrorretinografia de campo total bilateral utilizando eletrorretinógrafo Veris 2000, realizada em cão fêmea, Sem Raça Definida, 7 anos. 
média de 50 apresentações dos estímulos luminosos. O tempo médio de duração do ERG de campo total foi de 60 minutos, dentre os quais, 30 minutos foram para a adaptação ao escuro e 10 minutos para adaptação ao claro.

A amplitude da onda $A$ foi medida da linha de base até 0 pico negativo da onda $A$ e a onda $B$, foi medida do pico negativo da onda $A$ até o pico positivo da onda B. O tempo de culminação da onda $B$ foi medido a partir do início do estímulo até o pico máximo da respectiva onda (Fig.3). As amplitudes das respostas obtidas foram medidas em microvolts $(\mu \mathrm{V})$ e o tempo de culminação da onda B em milisegundos (ms). Todo o procedimento está demonstrado no Quadro 1.

\section{RESULTADOS}

Eletrorretinogama de campo total foi realizado com sucesso em 233 cães. Desses, 27,5\% não tinham raça definida (SRD). As raças examinadas incluíam principalmente Poodle $(31,3 \%)$ e Cocker Spaniel Inglês $(7,3 \%)$. Os cães examinados apresentavam catarata em diferentes estágios de maturação (100), eram diabéticos e apresentavam catarata madura ou hipermadura (72), apresentavam suspeita clínica de degeneração retiniana progressiva (26), síndrome da degeneração retiniana adquirida subitamente (três) ou eram considerados clinicamente normais quanto à função retiniana (32).

A sedação foi capaz de produzir boa imobilização do paciente sem rotacionar o bulbo ocular, o que permitiu adequada estimulação retiniana bilateral com auxílio do Ganzfeld. O bloqueio do nervo palpebral garantiu a obtenção de registros com baixo ruído, pois impossibilitou sua movimentação. Os cães aceitaram o procedimento devido à colocação dos eletrodos Burian Allen, que permaneceram sobre a córnea. Não se observou lesão corneal ou desconforto ocular após o exame.

Utilizando-se o Ganzfeld e o sistema eletrodiagnóstico Veris, obteve-se com sucesso as cinco respostas preconizadas pela ISCEV simultaneamente de ambos os olhos (Fig.4), conseguindo-se mensurar a amplitude das ondas A e B, o tempo de culminação da onda B, além do delta dos potenciais oscilatórios. Os registros dos cães considerados clinicamente normais quanto à função retiniana não apresentaram alterações capazes de atenuar as respostas do ERG. Comparando-se os demais registros a estes, observamos que: (1) cães com opacidade de meios, como catarata imatura, madura ou hipermadura, apre1sentaram atenuação na amplitude das respostas, principalmente de bastonetes, (2) em cães diabéticos houve diminuição dos potenciais oscilatórios, indicando lesão vascular nas camadas mais internas da retina, (3) em cães com suspeita de degeneração retiniana progressiva, amplitudes menores e a latência aumentada foram registradas na fase inicial da doença, já nas fases mais tardias, os registros apresentaram ausência de respostas, (4) os cães com suspeita de SARDS apresentaram ERG extinto, ou seja, não apresentaram ondas (Fig.5).

\section{DISCUSSÃO}

Com intuito de avaliar a função retiniana, o eletrorretinograma de campo total tem sido empregado com maior frequência, pois além de ser objetivo, detecta precocemente lesões nas camadas mais externas. A padronização do

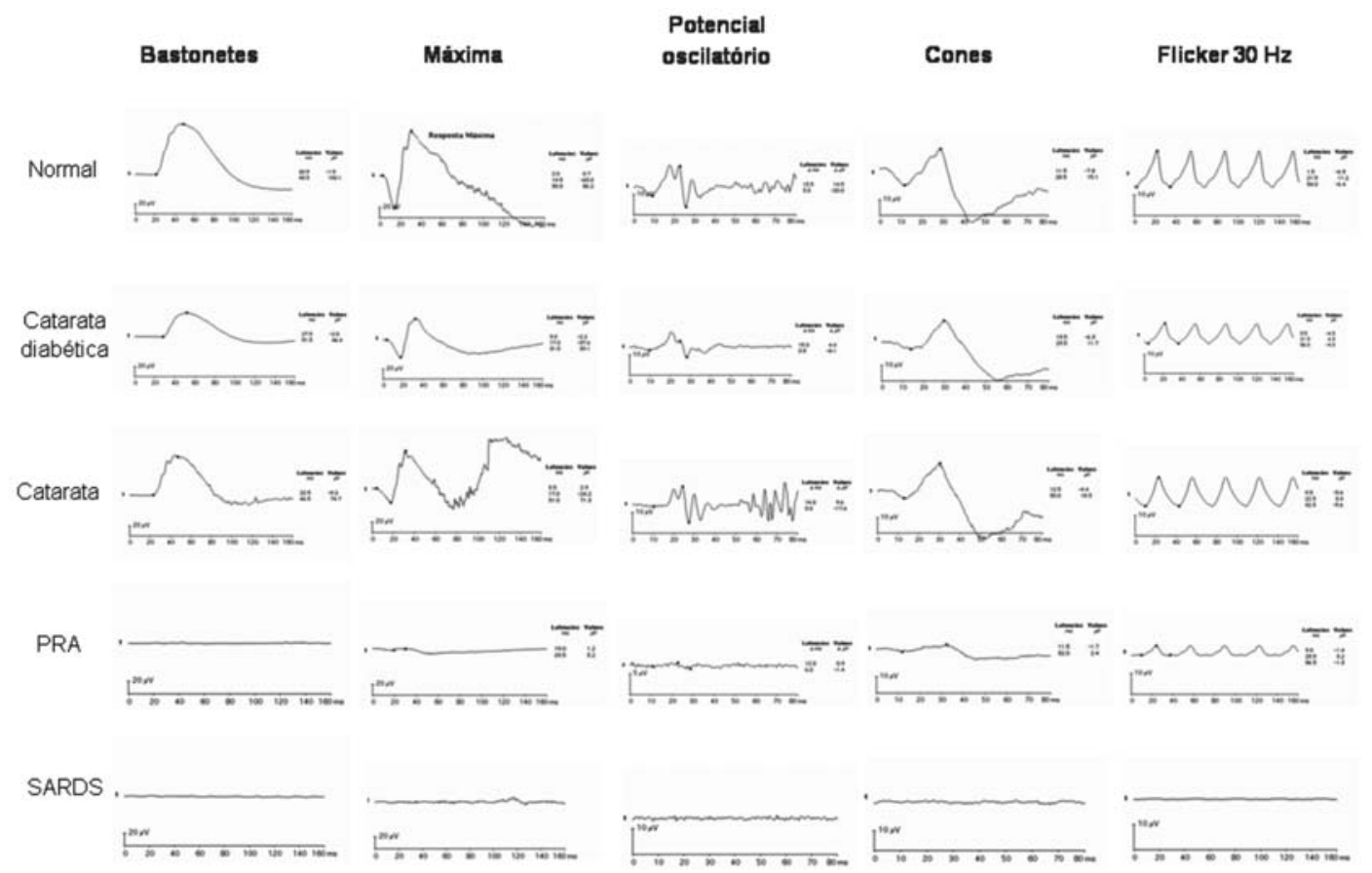

Fig.5. Comparação dos gráficos obtidos em cão normal e com diferentes oftalmopatias. Normal: fêmea, 8 anos, sem raça definida; Catarata diabética: fêmea, 8 anos, Weimaraner; Catarata: fêmea, 7 anos, sem raça definida; degeneração retiniana progressiva: fêmea, 4 anos, Poodle; síndrome da degeneração retiniana adquirida subitamente: macho, 7 anos, Schnauzer. 
exame é indispensável, pois vários são os fatores que alteram a resposta do exame, tais como: eletrodos, sedação, intensidade e frequência dos estímulos luminosos, dentre outros. Para a realização do exame, necessita-se a cooperação do paciente que deve permanecer imóvel. Apesar de não ser um procedimento doloroso, a imobilização do animais pode ser garantida com o uso de sedação ou anestesia e tem como objetivo evitar movimentos musculares, diminuir o estresse e permitir a colocação de forma adequada dos eletrodos, bem como o correto posicionamento do animal (Narfström 2006).

Dependendo da escolha do anestésico, a alteração do ERG pode ser mínima, como nos hipnóticos, ou importante quando utilizado barbitúricos ou anestésicos voláteis (Narfström 2006). O protocolo de sedação utilizando associação de sulfato de atropina, cloridrato de xilazina e butorfanol permitiu a obtenção de registros adequados, com baixo ruído. Para tal, o bloqueio do nervo palpebral foi indispensável, pois promoveu acinesia palpebral, uma vez que o sistema eletrodiagnóstico Veris não permite remover a interferência dos registros, quando da movimentação palpebral. Eletrorretinógrafos capazes de remover tal interferência dispensam este bloqueio.

Como vantagens neste protocolo de sedação, pode-se citar: maior segurança, praticidade e centralização do bulbo ocular, além da rápida recuperação do paciente, que ocorreu de forma tranquila e segura, isenta de agitação e tremores, quando comparada com anestesia geral. A sedação também permitiu realizar o procedimento de forma adequada no tempo proposto, que foi de aproximadamente $30 \mathrm{mi}-$ nutos. Ao término do exame, a maioria dos cães foi capaz de sair caminhando da sala de exame. Quando o cão é anestesiado, o bulbo ocular rotaciona, resultando em inadequada estimulação luminosa na retina. Assim, é necessário utilizar-se de bloqueio neuromuscular ou planos de anestesia mais profundos para sua centralização; em ambos os casos há necessidade de intubação traqueal.

A midríase de ambos os olhos foi obtida com instilação dos colírios de tropicamida $1 \%$ e fenilefrina $10 \%$ antes da adaptação ao escuro, o que resultou em boa dilatação pupilar, normalmente observada nos mamíferos em geral com duração de 3-5 horas. Objetivou-se obter diâmetro pupilar igual ou superior a $6 \mathrm{~mm}$ de diâmetro, o que nos permitiu a realização adequada do exame, uma vez que a retina pode ser amplamente estimulada pela luz. Não foi observada miose pós-sedação, o que pode acontecer em certos protocolos anestésicos (Petersen-Jones et al. 2006).

A adaptação ao escuro, como recomendada pela ISCEV, constou de 30 minutos e foi realizada em sala escura após a colocação de bandagem ao redor dos olhos, o que foi bem tolerado pelos pacientes. Adaptação mais demorada pode resultar em amplitudes maiores na fase escotópica do ERG e por isso deve ser evitada. O mesmo tempo de adaptação foi realizado por outros autores (Maehara et al. 2005).

A realização do exame foi feita em sala escura com auxílio de uma fonte de luz vermelha, para facilitar o correto posi- cionamento dos eletrodos sobre a córnea e a borda auricular e o adequado monitoramento da sedação durante a fase escotópica do ERG. Optou-se pela luz vermelha, pois esta não estimula os bastonetes, garantindo a resposta completa deste fotorreceptor ao flash gerado pelo Ganzfeld.

Sabe-se que respostas variadas são obtidas dependendo do eletrodo escolhido. Eletrodos não-corneais são menos estáveis que os corneais, porém, pelo menos um estudo (Kuze \& Uji 2000) demonstrou similaridade quanto à estabilidade ao compararem eletrodo DTL com Burian Allen em pacientes humanos. O uso do eletrodo DTL nos animais é mais difícil que no homem devido à dificuldade de fixação pela presença de pêlos nos cantos medial e lateral dos olhos.

Alguns autores (Maehara et al. 2005) utilizaram com sucesso o eletrodo monopolar LED (light-emitting diode) tipo lente de contato em ambos os olhos simultaneamente, criando condição similar de estimulação luminosa da retina quando comparado ao Ganzfeld. No referido estudo, mesmo utilizando cães da raça Beagle, foram necessários dois tamanhos diferentes de eletrodo. O eletrodo selecionado neste estudo foi o eletrodo Burian Allen, pois além de ser um eletrodo bipolar de contato corneal, capaz de registrar respostas com baixa interferência, também apresenta formato especular, promovendo abertura palpebral durante o exame. Utilizou-se três tamanhos diferentes do eletrodo: adulto, pediátrico e infantil, mas constatou-se a necessidade de diversos tamanhos para que pudessem ser posicionados em diversas raças. Lesão corneal resultante do exame pode ser evitada protegendo a córnea com metilcelulose $2 \%$ acrescida de colírio anestésico e não foi observada em nenhum paciente deste estudo. Com o auxílio do Ganzfeld, que apesar de ser dispendioso e de difícil instalação (Maehara et al. 2005), conseguiu-se estimular os dois olhos de forma uniforme e simultânea.

Nesta padronização, o protocolo do ERG de campo total utilizado foi o mesmo recomendado pela ISCEV para o homem, constituído de cinco respostas. O protocolo sugerido pela ECVO em 2002, consiste de quatro respostas (Narfström et al. 2002), não registrando-se os potenciais oscilatórios, que são úteis na avaliação de distúrbios vasculares na retina, como na retinopatia diabética, por isso, optou-se por seguir o protocolo padrão da ISCEV.

Utilizando-se o sistema eletrodiagnóstico Veris, registrou-se gráficos com baixo ruído e que apresentaram as ondas A e B com picos nitidamente detectáveis e mensuráveis. Os registros foram, portanto, considerados adequados para a determinação das amplitudes das ondas, os tempos de culminação da onda $B$, além do delta dos potenciais oscilatórios permitindo o diagnóstico das alterações retinianas observadas com confiabilidade.

A interpretação do ERG deve ser feita levando-se em consideração, além da opacidade de meios e da doença que o animal apresenta, a espécie, a raça e a idade. Quanto mais jovem for o paciente, maiores são as amplitudes das respostas, por outro lado, quanto mais velho, menores são as amplitudes, semelhante ao que ocorre no ERG em humanos (Narfström 2006). 
Ausência completa das ondas $A$ e $B$, ou seja, ERG extinto, significa lesão completa dos fotorreceptores, do complexo Müller e células bipolares, o que é sugestivo de degeneração retiniana hereditária, descolamento antigo e total da retina (Gonçalves et al. 2000), SARDS, dentre outros (Cullen \& Grahn 2002).

\section{CONCLUSÕES}

Neste estudo, observamos diferentes padrões de registros, demonstrando que este protocolo é capaz de identificar, com segurança, diferentes alterações retinianas, tais como: degeneração retiniana progressiva, retinopatia diabética e SARDS. Observou-se também a diferença entre a obtenção dos registros em cães que apresentavam ou não opacidade de meios (catarata imatura, madura ou hipermadura), devido a baixa intensidade luminosa incapaz de estimular adequadamente a retina.

Em todos os cães empregou-se o mesmo protocolo na realização do exame, utilizando mesmo tempo de adaptação ao escuro e ao claro, mesmos eletrodos, mesmo procedimento para a sedação e mesmo aparelho. A confiabilidade e reprodutibilidade desta padronização foi demonstrada com a obtenção de registros de ótima qualidade utilizando protocolo padrão da ISCEV nos cães examinados.

Sugere-se a padronização dos valores dos registros, seguindo o padrão de ERG de campo total aqui demonstrado, nas diferentes espécies e raças, com diferentes idades, para as diversas oftalmopatias, pois o ERG de campo total tornou-se exame fundamental na rotina oftalmológica. Assim, sua padronização é indispensável quando se objetiva comparar resultados de laboratórios distintos.

Agradecimentos.- À Fundação de Amparo à Pesquisa do Estado de São Paulo (FAPESP, Proc. 02/08051-9) pelo auxílio financeiro e ao Serviço de Eletrofisiologia Visual do Departamento de Oftalmologia da Universidade Federal de São Paulo (Unifesp) na implantação do Setor de Eletrofisiologia do Laboratório de Investigação em Oftalmologia Comparada da FMVZ-USP.

\section{REFERÊNCIAS}

Beeler P., Barthelmes D., Sutter F.K., Helbig H. \& Fleischhauer J.C. 2007. Comparison of performance and patient satisfaction of two of ERG electrodes. Klin. Monatsbi. Augenheilkd. 224(4):265-268.

Birch D.G. 1989. Clinical electroretinography: Assessment of visual function for the clinician. Ophthalmol. Clin. North Am. 2:469-98.

Birch D.G \& Anderson J.L. 1992. Standardized full-field electroretinography: Normal values and their variation with age. Arch. Ophthalmol. 110:1571-6.

Català J. \& Castany M. 2005. Pruebas electrofisiológicas: qué, cuando, como y por qué? (2 $2^{\mathrm{a}}$ parte). Annals dфcOftalmologia 13(2):76-90.

Cullen C.L. \& Grahn B.H. 2002. What are your clinical diagnosis, lesion localization, and differential diagnoses? Can. Vet. J. 43(9):729-730.

Dantas A.M. 1995. Eletrorretinografia, p.141-225. In: Dantas A.M., Costa J.G.C., Neto L.P., Yamane R. \& Elias C.A. (Eds), Eletrofisiologia Ocular. Cultura Médica, Rio de Janeiro.

Gonçalves E.R., Fernandes M.L., Takahashi W.Y. \& Suzuki H. 2000. Eletrofisiologia ocular, p.185-225. In: Conselho Brasileiro de Oftalmologia \& Sociedade Brasileira de Retina e Vítreo (Eds), Retina e Vítreo: Clínica e cirurgia. Roca, São Paulo.
Gum G.G. 1980. Electrophysiology in veterinary ophthalmology. Vet. Clin. North Am., Small Anim. Pract. 10:437-454.

Hendrix D.V.H. \& Sims M.H. 2004. Electroretinography in Hispaniolan Amazon parrot (Amazona ventralis). J. Avian Med. Surg. 18:89-94.

Hérbert M., Lachapelle P. \& Dumont M. 1996. Reproducibility of electroretinograms recorded with DTL electrodes. Documenta Ophthalmologica 91:333-342.

Hurn S.D., Hardman C. \& Stanley R.G. 2003. Day-blindness in three dogs: Clinical and electroretinographic findings. Vet. Ophthalmol. 6(2):127-130.

Komaromy A.M., Smith P.J. \& Brooks D.E. 1998. Electroretinography in dogs and cats. Part I. Retinal morphology and physiology. Compend. Cont. Educ. 20:343-354.

Komaromy A.M., Andrew S.T., Sapp Jr. H.L., Brooks D.E. \& Dawson W.W. 2003. Flash electroretinography in standing horses using the DTL ${ }^{\mathrm{tm}}$ microfiber electrode. Vet. Ophthalmol. 6:27-33.

Kommonen B. \& Raitta C. 1987. Electroretinography in Labrador Retrievers given ketamine-xylazine anesthesia. Am. J. Vet. Res. 48:1325-1331.

Kuze M. \& Uji Y. 2000. Comparison between Dawson, Trick, and Litzkow electrode and contact lens electrode used in clinical electroretinography. Japan. J. Ophthalmol. 44:374-380.

Maehara S., Itoh N., Wakaiki S., Tsuzuki K., Seno T., Kushiro T., Yamashita K., Izumisawa Y. \& Kotani T. 2005. Electroretinography using contact lens electrode with built-in light source in dogs. J. Vet. Med. Sci. 67(5):509-514.

Marmor M.F., Arden G.B., Nilsson S.E.G. \& Zrenner E. 1989. Standard for clinical electroretinography. Arch. Ophthalmol. 107:816-819.

Marmor M.F. \& Zrenner E. 1999. Standard for clinical electroretinography (1999 update). Documenta Ophthalmologica 97:143-156.

Marmor M.F., Holder G.E., Seelige R.M.W. \& Yamamoto S. 2004. Standard for clinical electroretinography (2004 update). Documenta Ophthalmologica 108:107-114.

Narfström K., Ekesten B., Rosolen S.G., Spiess B.M., Percicot C.L. \& Ofri R. 2002. Guidelines for clinical electroretinography in the dog. Documenta Ophthalmologica 105:83-92.

Narfström K. 2006. Electroretinographic testing in larger animals, p.923933. In: Heckenlively J.R. \& Arden G.B. (Eds), Principles and Practice of Clinical Electrophysiology of Vision. $2^{\text {nd }}$ ed. Mit, Cambridge.

Narfström K., Wrigstad A., Ekesten B. \& Bergss L.A. 2007. Neuronal ceroid lipofuscinosis: Clinical and morphologic findings in nine affected Polish Owczarek Nizinny (PON) dogs. Vet. Ophthalmol. 10(2):111120.

Paranhos F.R.L., Ávila M.P., Paranhos A. \& Cialdini A.P. 1997. Estudo estatístico de valores normais do eletrorretinograma: contribuição à padronização do exame. Arqs Bras. Oftalmol. 60:278-284.

Petersen-Jones S., Tuntivanich N., Montiani-Ferreira F. \& Khan N. 2006. Electroretinograms of dog and chicken, p.911-921. In: Heckenlively J.R. \& Arden G.A. (Eds), Principles and Practice of Clinical Electrophysiology of Vision. $2^{\text {nd }}$ ed. Mit, Cambridge.

Rubin L.F. 1971. Clinical features of hemeralopia in adult Alaskan Malamute. J. Am. Vet. Med. Assoc. 158(10):1696-1698.

Safatle A.M.V., Salomão S., Berezovsky A., Sacai P., Fantoni D., Yasbek K. \& Barros P.S.M. 2005. Retinal degeneration in a Pit Bull dog: Electroretinographic findings. Archs Vet. Sci. 10(2):119-124.

Salomão S.R. 2002. Eletrofisiologia visual nas uveítes, p.162-189. In: Abreu M.T. (Ed.), Inflamações Oculares. Roca, São Paulo.

Sims M.H. 1999. Electrodiagnostic evaluation of vision, p.483-507. In: Gelatt K.N. (Ed.), Veterinary Ophthalmology. $3^{\text {rd }}$ ed. Lippincott, Williams and Wilkins, Philadelphia.

Tzecov R. \& Arden G.B. 1999. The electroretinogram in diabetic retinopathy. Survey of Ophthalmology 44(1):53-60. 